The $7^{\text {th }}$ International Conference on Nuclear Microprobe Technology and Applications, Bordeaux, France, Sept 2000

\title{
Luminescent Layers for Ion-Photon Emission Microscopy
}

C. Yang ${ }^{a}$, B. L. Doyle ${ }^{b}$, P. Rossi ${ }^{c}$, M. Nigam ${ }^{a}$, M. El Bouanani ${ }^{a}$, J. L. Duggan and F. D. McDaniel ${ }^{a}$

${ }^{a}$ Ion Beam Modification and Analysis Laboratory, Department of Physics, University of North Texas, Denton, Texas 76203, USA

${ }^{b}$ Ion Beam Materials Research Laboratory, MS 1056, PO Box 5800 Sandia National Laboratories, Albuquerque, NM 87185, USA

${ }^{c}$ Dipartimento di Fisica dell'Universita and INFN, via Marzolo 8, I-35131 Padova, Italy

\begin{abstract}
Ion Beam Induced Luminescence (IBIL) combined with Ion Beam Induced Charge Collection (IBICC) is applied in a quantitative study of the IBIL generation yield and detection efficiency for several plastic phosphor materials. The main purpose of this study is to search for strongly luminescent materials that can be used to easily coat samples to be studied with Ion Photon Emission Microscopy (IPEM). A special focus is given to plastic scintillation materials because thin films are easily prepared, and such films have already been used for single event triggering. The emission yield was found to be low for typical Bicron plastic phosphors (only $\sim 70$ photons/ion/micron). The total collection, transmission and photon detection efficiency of the optical microscope used in this study was determined to be only $\sim 0.00003$. For thin film plastic phosphors $\sim 20$ microns thick, the detection efficiency was only 0.04 photons/ion. This means that using these plastics, IPEM would need to be performed with $\sim 20 \mathrm{x}$ more beam fluence to obtain data, such as IBICC, similar to a standard scanned nuclear microprobe. Improvements are discussed.
\end{abstract}




\section{DISCLAIMER}

This report was prepared as an account of work sponsored by an agency of the United States Government. Neither the United States Government nor any agency thereof, nor any of their employees, make any warranty, express or implied, or assumes any legal liability or responsibility for the accuracy, completeness, or usefulness of any information, apparatus, product, or process disclosed, or represents that its use would not infringe privately owned rights. Reference herein to any specific commercial product, process, or service by trade name, trademark, manufacturer, or otherwise does not necessarily constitute or imply its endorsement, recommendation, or favoring by the United States Government or any agency thereof. The views and opinions of authors expressed herein do not necessarily state or reflect those of the United States Government or any agency thereof. 


\section{DISCLAIMER}

Portions of this document may be illegible in electronic image products. Images are produced from the best available original document. 


\section{Introduction}

Knowledge of the IBIL yield and optical detection efficiency of a wide variety of thin film phosphors is crucial to the success of a new nuclear emission microscopy: Ion Photon Emission Microscopy (IPEM), currently under development at Sandia [1]. We hope to develop IPEM as a new tool for performing single ion effects microscopies without beam focusing, and potentially with a radioactive source instead of an accelerator. These thin films must be deposited or applied to the surface of samples such as microelectronic integrated circuits (ICs) to enhance the production of ion-induced photons. These photons are then projected with a high magnification lens system onto a position sensitive detector (PSD) that is sensitive to single photons. The efficient generation, transmission and detection of these photons is required using the IPEM technique to determine the arrival position of each ion which strikes the sample. The utility of IPEM therefore hinges on finding an optimum lumenescent layer.

It is well known that inorganic ceramic phosphors have the greatest lumenecence. Unfortunately, it is very challenging to make and apply extremely thin homogenous phosphors layers (a few microns thick) on top of ICs from such materials since they usually must be processed at very high temperature. Other standard deposition techniques such as sedimentation lead to extremely rough layers that are not suitable for IPEM due to light scattering effects.

Plastic phosphors have several advantages over inorganic ceramic materials. They usually have a fast decay time (a few ns to tens of ns); they are clear and smooth (reducing or even eliminating light scattering); they are easily made thin and self-supporting; and such plastic scintillators are already commercially available.

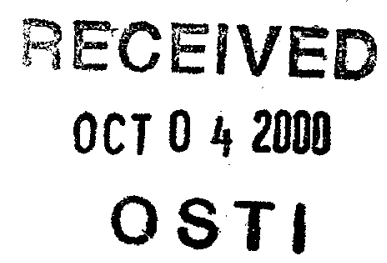


This paper reports the first in a series of experiments aimed at finding an optimum highly lumenescent thin film material for IPEM. The first materials studied were organic phosphors.

\section{Instrumentation}

The nuclear microprobe system [2] used in this study is installed on the 3MV NEC 93DH-2 Pelletron tandem accelerator. A source of negative ions by cesium sputtering (SNICS) provides a wide variety of ion species, with $\mathrm{H}$-ions being used here. Alpha particles are provided by the second ion source, the NEC Alphatross Ion Source, which can produce negative helium ions for the injection to the tandem accelerator.

The nuclear microprobe chamber is equipped with a high quality optical microscope (SM-OM40, JEOL USA, Inc.). This microscope was originally designed for scanning electron microscopy (SEM) for the observation of micrographic images with a very high magnification (x300) and a viewing field of 650 microns in diameter. The optical microscope has an objective lens in a retractable long arm. The objective lens is a type of refracting lens with a hole drilled through its center through which the ion beam passes during nuclear microprobe experiments (see Fig. 1). The optical microscope has an easy working distance of $5 \mathrm{~mm}$ and numeral aperture (N.A.) about 0.3 .

With a large magnification, the microscope is ideal for viewing very small features (approximately one-micron resolution). The monocular eyepiece of the microscope can be easily replaced by a CCD camera or a luminescence detector such as PMT (photomultiplier tubes) for Ionoluminescence applications. In this work, a PMT-based integrated photon counting head (Hamamatsu Model H5920-01) is used for a single photon counting detector. This luminescence detector has an output of 3.3 volts pulse height and $25 \mathrm{~ns}$ pulse width. The highest sensitivity of the detector is at about $400 \mathrm{~nm}$ wavelength with about 
$16 \%$ quantum efficiency. The dark current of the photon counting head is about $1-2 \mathrm{cps}$, after stored in dark room more than $24 \mathrm{hrs}$.

In this study, a PIN-diode (Hamamatsu, model S1223) is used to detect and count the incoming particles which excite the IBIL photons. Thick and thin plastic phosphors are laid on the top surfaces of the S1223 PIN-diodes. The layout of the ion-sample geometry and the data acquisition system in Denton Nuclear Microprobe is also shown schematcially in Figure 1. The experiments to determine the detected photon/ion efficiency proceeds just like an IBICC experiment on the PIN diode, where in addition, the IBIL signal is measured at the same time.

\section{Samples preparation}

\section{Thick samples:}

Thick samples of the Bicron series: BC-400, BC-404, BC-408, and BC-430 were laid on the top surfaces of the PIN-diodes, as shown in Figure 2. This is for the investigation of the IBIL efficiency on original samples without any processing. Small pieces of the plastic scintillators were cut from the polished surface side of the original material purchased from BICRON ${ }^{\mathrm{TM}}$ and glued to a surface of a PIN-diode.

\section{Thin samples:}

The recipe for creating thin self-supporting blades using commercially available plastic blocks like Bicron's has been described in reference [3], in which further literature on this subject can be found. The method consists of diping some plastic powder, obtained by scraping the block, in toluene; waiting a few hours until the powder is fully dissolved, stirring to get an uniform solution; removing the less dense part of solution and spreading the remaining part over a level glass surface; and finally waiting few hours for the toluene to evaporate and the formation of a solid film, which can be easily removed after 
immersion in a glass of distilled water. The quantity of powder and toluene are not critical except that ther must be enough toluene to dissolve all of the powder. To get a film of variable thickness, the glass surface can be slightly tilted, and the film in the lower part will become thicker. This is a way to produce blades of different thickness during one deposition run.

\section{The IBIL efficiency of plastic scintillators}

The maximum size of the sample imaging area is limited not by beam scanning, but by the size of the hole in the objective lens of the OM-40 optical microscope. A scanning test was carried out on a sample made of a PIN-diode with a 400 mesh gold grid mounted on its surface. As shown in the Figure 3 (a), the maximum scanning range can be made about $640 \mu \mathrm{m}$ without serious distortion. Figure 3 (b) displays a higher magnification IBIL image from a scan over a sample made of $\mathrm{BC} 400$ scintillator with a 1000 mesh copper grid mounted on its surface. In determining the photon/ion detection efficiency it is important that the data analysed from these combined IBICC and IBIL measurements comes from the central region of the image to avoid edge effects which can attenuate the photon flux.

Large scans were made of the thick samples mounted on PIN-diodes. The scans were made to include 1) an area for IBICC analysis of the PIN-diode surface which remained uncovered and 2) part of sample area covered by the thick plastic which produced an IBIL signal. A typical IBIL and IBICC image for such a thick plastic phosphor is displayed in Figure 4. The quantitative information of ions/pixel is extracted from the IBICC image area where the PIN-diode is not covered by the thick sample. Similarly, the quantitative information on photons/pixel is extracted from the IBIL image area where the PIN-diode is covered by the thick sample. Therefore, by the combination of this information, the IBIL efficiency in term of photons/ion can be easily derived. These detected photons/ion 
experiments were carried out using three ion beams: $3.0 \mathrm{MeV} \mathrm{H}, 6.0$ and $9.0 \mathrm{MeV} \mathrm{He}$ and four Bicron samples: BC 400, BC 404, BC-408 and BC 430. The results are listed in Table 1.

Two thin (18 and 21 microns) BC-408 samples were also investigated using alpha particles at energies of $3.9,6.0$ and $9.0 \mathrm{MeV}$. The detected photons/ion results of these measurements are also listed in Table 2. The typical IBIL and IBICC images for the thin plastics are displayed in Figure 5; where the scan size is about $750 \mu \mathrm{m}$. The left round edge shape of the images is due to beam clipping by the edge of the hole in the objective lens.

\section{Birks IBIL Intensity Theory}

The IBIL mechanism in plastic material is molecular in nature. (***I have no idea what this means?) The non-linearity of the light output from organic material is very pronounced and became very noticeable for heavy ions at low energy. The light output per unit length, $d L / d x$ (photons/micron), is well described by Birks formula [4]:

$$
\frac{d L}{d x}=\frac{F \cdot A_{a n t h} \cdot\left(\frac{d E}{d x}\right)}{1+\beta \cdot\left(\frac{d E}{d x}\right)} ; \quad A_{a n t h}: \quad \begin{aligned}
& \text { absolute scintillation efficiency of anthracene } \\
& \text { it is about } 1.67 \mathrm{E}+04 .
\end{aligned}
$$

When

$$
\beta \cdot\left(\frac{d E}{d x}\right)>>1
$$

which is the case for He ions and heavier, there is saturation:

$$
\frac{d L}{d x} \approx \frac{F \cdot A_{\text {anih }}}{\beta}
$$

The IBIL production in the excitation volume can be derived from the detected photons:

$$
\frac{d L}{d x}=\frac{L_{\mathrm{det}}}{f_{A} \cdot f_{\Omega} \cdot f_{T} \cdot f_{Q} \cdot X}
$$


Or

$$
\frac{d L}{d x}=\frac{L_{\mathrm{det}}}{f_{\text {all }} \cdot X} \quad f_{\text {all }}=f_{A} \cdot f_{\Omega} \cdot f_{T} \cdot f_{Q}
$$

The experimental data from table 1 can been reproduced using the Birks formula listed above (and assuming the anthracene value $\beta=117$ ); the data fitting gives the wholedetection-efficiency factor as: $f_{a l l}=4.0$ E-5. This suggests the optical microscope transmission rate is about $4 \%$. The overall IBIL yield is only $60-80$ photons/ions/micron for all of these phosphors.

The experimental data from the thin samples (data listed in Table 2) has also been fit using Birk's formula; however, the fitting tends to give lower whole-detection-efficiency factor: $f_{a l}=3.0$ E-5. This may suggest that the thin sample preparation procedure may change the properties of the plastic scintillators, resulting in lower IBIL yield efficiency.

\section{Conclusions}

The IBIL efficiency of some typical organic phosphors is quantified through the combined use of IBIL and IBICC in the nuclear microprobe. The organic scintillation material studied are ideal for fabrication of homogenous thin layers for the IPEM application; however, their IBIL yield efficiency was measured to be only $\sim 70$ photons generated per ion per micron. Using such phosphors as surface layers for IPEM will require a high detection efficiency for photon collection, transmission and recording.

This was found not to be the case using the OM-40 microscope-PMT system which had a total detection efficiency was only $\sim 0.004 \%$ : light collection $=\sim 1 \%$, microscope transmission $=\sim 4 \%$, quantum efficiency of the PMT $=\sim 10 \%$. Nothing can be done regarding the low efficiency of the collection and detection efficiency. The low 
transmission efficiency of the microscope could be due to several factors including reflection losses at each optical element, and absorption by the glasses used to form these elements.

Unless plastics with greater luminescence are found, a more efficient photon projection system must be devised if these materials are to be used for IPEM coatings. One approach would be to increase the collection efficiency by reducing the lens-sample distance, and minimize both the reflection losses (i.e. reduce the number of optical elements in the microscope) and the absorption losses (i.e. reduce the optical path through these elements). This could be accomplished in principle by using a single short-focallength lens projector. The only other element in this system would be the PSD placed at some distance from the lens. This could result in a high magnification system, with good collection and minimal optical losses. We estimate that the system efficiency could be increased at least a factor of 20 , and that the detected photons/ion efficiency could approach 1, which, while not required for IPEM, is certainly desireable.

\section{Acknowledgements:}

The work is supported in part by NSF, the State of Texas Advanced Technology Program, and the Robert A. Welch Foundation. Work at Sandia supported by the U.S. DoE under contract DE-AC04-94AL85000.

\section{Reference:}

[1] B.L. Doyle and et al.. This proceedings.

[2] F. D. McDaniel, J. L. Duggan, C. Yang, B.N. Guo, M. El Bouanani, and M. Nigam, this proceedings.

[3] D. Bernardi, G.P. Egeni, F. Parere, M. Pegoraro, P. Rossi, V. Rudello, H. Somacal, E. Vittone, M. Viviani, Nucl. Instr. and Meth. B 152 (1999) 377-385. 
[4] J.B. Birks, Proc. Phys. Soc. A64, 874 (1951).

[5] B.G. Yacobi and D.B. Holt, in "Cathodoluminescence Microscopy of Inorganic Solids", Plenum Press, New York and London, 1999. 


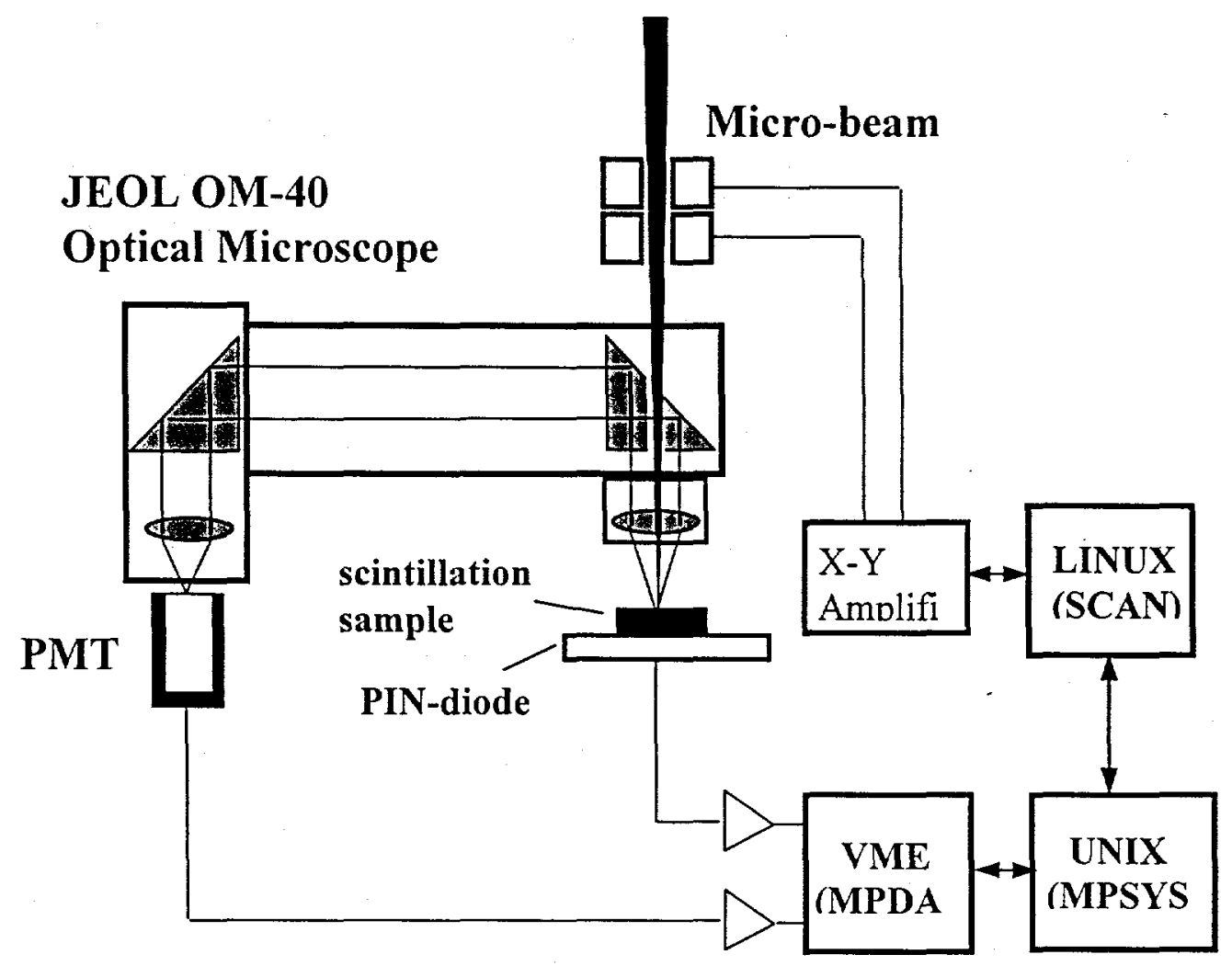

Figure 1. The layout of the sample stage and the data acquisition system in Denton Nuclear Microprobe. 
Figure 3. (a) An IBIC image of a large scan over a 400 mesh grid on a PIN-diode. It is possible to make a large scan over area about 640 um without cutting off the beam. 3.9 $\mathrm{MeV}$ alpha beam is applied. (b) IBIL image of a 1000 mesh grid over BC-400 plastic scintillation material.
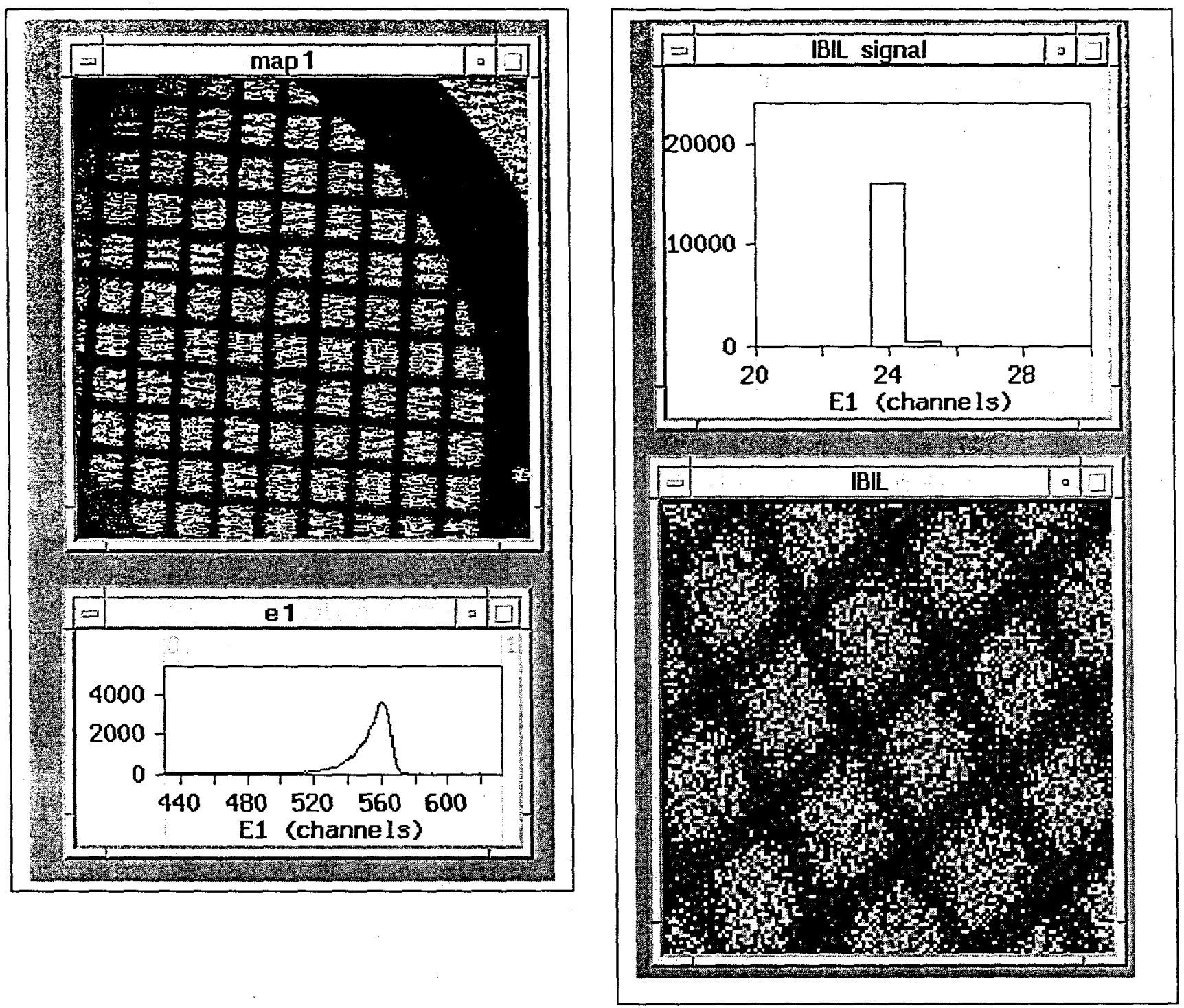


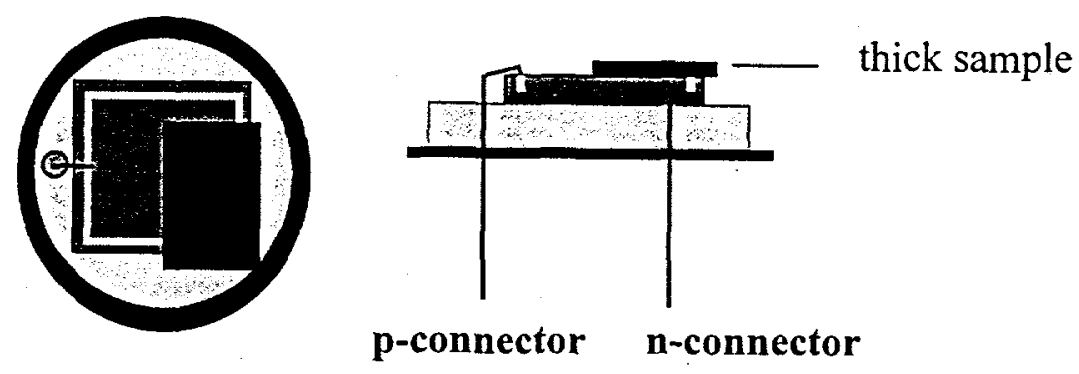




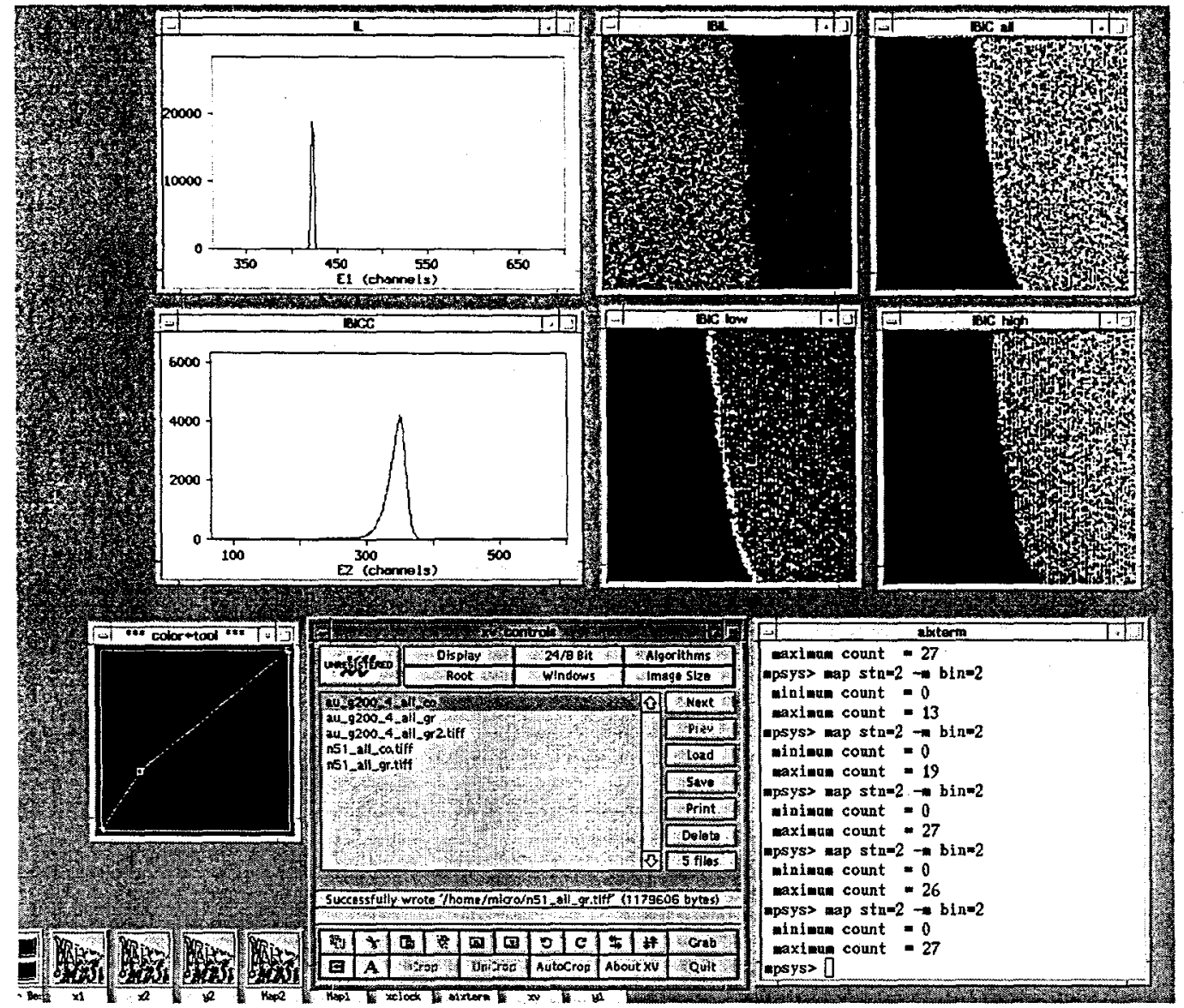

Figure 4. The IBIL and IBICC images over a scanned area. A thick piece of BC-404 plastic scintillation material is analyzed for the investigation of the IBIL production efficiency using $9 \mathrm{MeV}$ alpha beam. The sample-uncovered area in the IBICC image gives an information on ions/pixel. The sample-covered area in IBIL image gives the information on photons/pixel. By the combination of the two maps' information, the IBIL efficiency in term of photons/ion can be derived. 


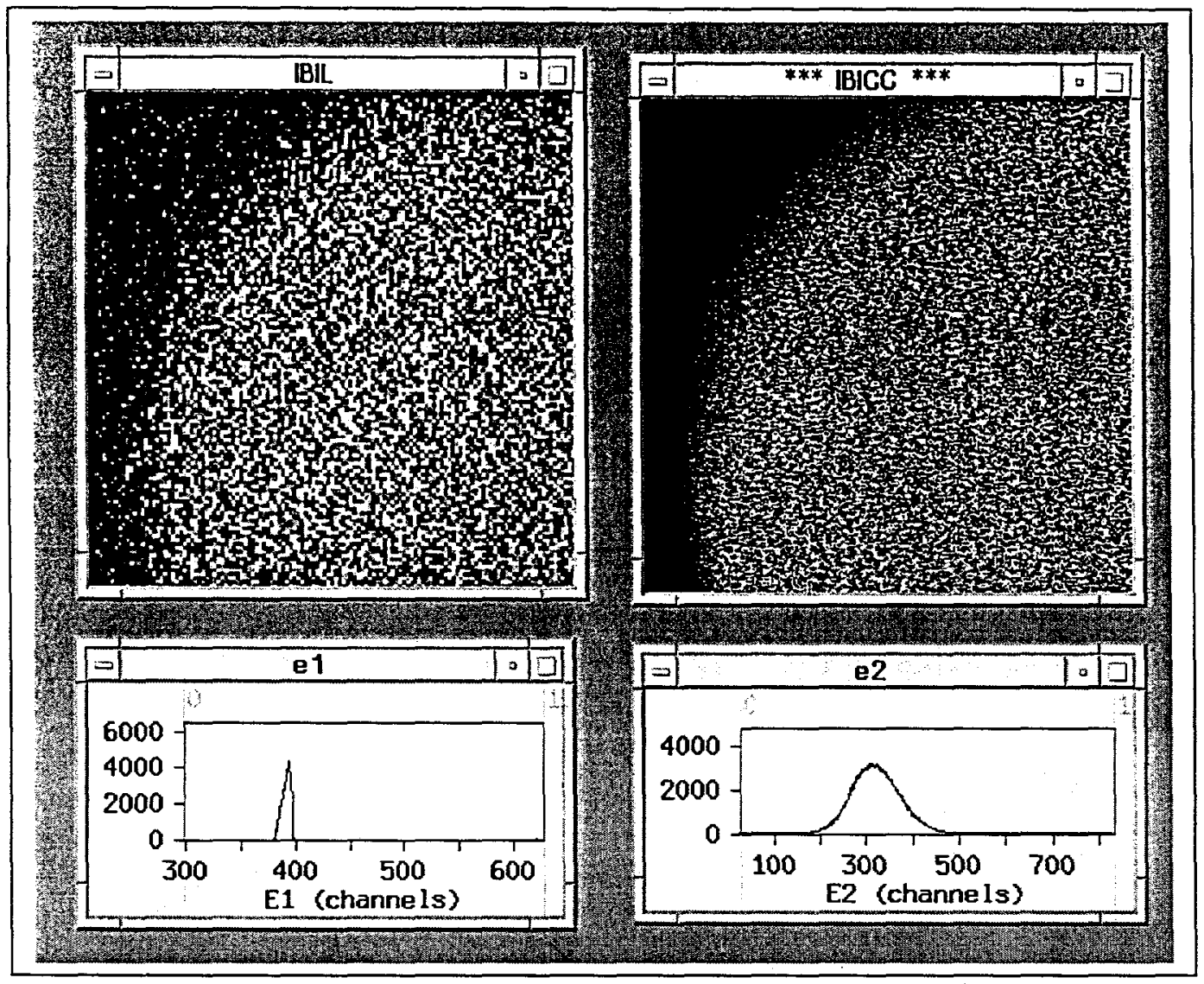

Figure 5. The IBIL and IBICC images of a thin BC-408 scintillation sample (18 um thick). The scanned area is over $800 \mathrm{um}$ in diameter. The left round edge shape of the images is due to the cutting off the scanned beam by the hole edge of the objective lens. $3.9 \mathrm{MeV}$ alpha beam is applied in the study. 
Table 1. Detected photons/ion for the plastic scintillation thick samples using protons and alpha particles. $\left(A_{a n t h}=1.65 \mathrm{E}+4, f_{A}=1, f_{Q}=0.1, f_{\Omega}=0.01\right)$

\begin{tabular}{|c|c|c|c|c|c|c|}
\hline $\begin{array}{c}\text { Ion Energies } \\
\text { Thick samples }\end{array}$ & $\begin{array}{c}3.0 \mathrm{MeV} \mathrm{H} \\
\mathbf{L}_{\text {det }} \\
\text { (photon/ion) }\end{array}$ & $\begin{array}{c}6.0 \mathrm{MeV} \mathrm{He} \\
\mathbf{L}_{\text {det }} \\
\text { (photon/ion) }\end{array}$ & $\begin{array}{c}9.0 \mathrm{MeV} \mathrm{He} \\
\mathbf{L}_{\text {det }} \\
\text { (photon/ion) }\end{array}$ & $\begin{array}{c}\mathbf{n} \\
\text { (refr. index) }\end{array}$ & $\mathbf{F}$ & $\begin{array}{c}\text { Wavelength } \\
\text { (nm) }\end{array}$ \\
\hline BC400 & $22.50 \%$ & $18.20 \%$ & $27.40 \%$ & 1.58 & 0.65 & 423 \\
\hline BC404 & $29.20 \%$ & $26.40 \%$ & $36.20 \%$ & 1.58 & 0.68 & 408 \\
\hline BC408 & $29.30 \%$ & $23.80 \%$ & $35.10 \%$ & 1.58 & 0.64 & 425 \\
\hline BC430 & $20.90 \%$ & $17.10 \%$ & $26.80 \%$ & 1.58 & 0.45 & 580 \\
\hline
\end{tabular}

Table 2. Detected photons/ion from thin samples using alpha particles. $\left(A_{\text {anth }}=1.65 \mathrm{E}+4, f_{A}=1, f_{Q}=0.1, f_{\Omega}=0.01\right)$

\begin{tabular}{|c|c|c|c|c|}
\hline $\begin{array}{c}\text { Ion Energies } \\
\text { Thin samples }\end{array}$ & $\begin{array}{c}3.9 \mathrm{MeV} \mathrm{He} \\
\text { det. (pho/ion) }\end{array}$ & $\begin{array}{c}6.0 \mathrm{MeV} \mathrm{He} \\
\text { det. (pho/ion) }\end{array}$ & $\begin{array}{c}9.0 \mathrm{MeV} \mathrm{He} \\
\text { det. (pho/ion) }\end{array}$ & $\begin{array}{c}\mathbf{N} \\
\text { (refr. index) }\end{array}$ \\
\hline $\begin{array}{c}\text { BC408 }(18 \mathrm{~mm}) \\
\text { Processed 1 }\end{array}$ & $4.11 \%$ & $4.60 \%$ & $4.30 \%$ & 1.58 \\
\hline $\begin{array}{c}\text { BC408 }(21 \mathrm{~mm}) \\
\text { Processed 2 }\end{array}$ & $4.15 \%$ & $4.70 \%$ & $4.60 \%$ & 1.58 \\
\hline
\end{tabular}

